\title{
Diagnostic du sexe des embryons bovins par biologie moléculaire
}

\author{
M Kirszenbaum1, C Cotinot2, M Leonard2, \\ $M$ Vaiman', M Fellous ${ }^{3}$
}

\author{
Avec la collaboration technique de S Ruffini ${ }^{2}$ \\ 1 Laboratoire de Radiobiologie Appliquée, CEA-IPSN-DPS-SPE, 78350 Jouy-en-Josas; \\ 2 Laboratoire de Biologie Cellulaire et Moléculaire, INRA, 78350 Jouy-en-Josas; \\ 3 Institut Pasteur, INSERM U276, 75015 Paris, France
}

(28 réunion de la Société Française pour l'Étude de la Fertilité; Paris, 19-21 octobre 1989)

\begin{abstract}
Résumé - Grâce à la construction d'une bibliothèque génomique bovine enrichie en séquences mâles, nous avons obtenu une sonde spécifique du chromosome $Y$, répétée environ 2000 fois dans le génome mâle des animaux du genre Bos et Bison. Pour être compatible avec le transfert et/ou la congélation des embryons, la détermination du sexe doit être effectuée sur une biopsie de 10 à 20 cellules prélevée sur un blastocyste de $7 \mathrm{j}$. L'hybridation in situ avec la sonde BC 1.2 biotinée suivie de la révélation immunocytochimique a permis de visualiser le signal d'hybridation sur le noyau de chaque cellule mâle. Cependant, à cause de ces nombreuses étapes, cette technique est apparue difficile d'application en routine. Pour cette raison, nous avons développé la technique de l'amplification enzymatique dirigée (PCR). A partir de la séquence BC 1.2, nous avons déterminé les amorces et les sondes dans le but d'amplifier des séquences spécifiques du chromosome Y. Cette technique, facile d'utilisation et d'automatisation, a permis d'obtenir un fort signal d'hybridation spécifique du mâle à partir de l'ADN génomique et des cellules embryonnaires.
\end{abstract}

sexage / embryon bovin / chromosome $Y$ / hybridation in situ / amplification enzymatique dirigée

Summary - Sexing of bovine embryos. Thanks to a bovine genomic library enriched with $Y$ chromosome-specific sequences, a probe specific to this chromosome of genera Bos and Bison and repeated on about 2000 copies in the male genome was isolated. To be compatible with embryo transfer and/or freezing, sex determination has to be done on a 10-20 cell embryo biopsy from a day-7 bovine blastocyst. The in situ hybridization with biotinylated BC 1.2 probe and immunocytochemical revelation permitted the visualization of the hybridization signal on the nucleus of each cell. Because of the numerous steps required, this technique was difficult to apply routinely. For that reason we worked out the polymerase chain reaction technique. From the BC 1.2 sequence, we determined several oligonucleotide primers and probes with the aim of amplifying the $Y$ chromosomespecific sequences. This technique, which is easy to perform and to automate, enabled us to obtain a strong male-specific hybridization signal on genomic DNA and embryo cells.

sexing / bovine embryo / Y chromosome / in situ hybridization / PCR amplification 


\section{INTRODUCTION}

Depuis la généralisation de l'insémination artificielle chez les bovins. le transfert d'embryons représente la seconde étape décisive dans l'évolution des techniques de reproduction de cette espèce. Parmi les multiples applications que l'on peut attendre du transfert, citons la possibilité d'accroître de manière significative le nombre de descendants femelles d'une vache sélectionnée par son haut potentiel génétique. Une vache ayant en moyenne 1,5 à 2 descendantes au cours de sa vie, les traitements hormonaux de superovulation associés au transfert d'embryons permettraient de décupler ce nombre et ceci d'autant plus facilement que le sexe des embryons serait connu. De même, la possibilité pour les éleveurs de faire naître d'une façon régulière des veaux de sexe désiré représente un gain de productivité non négligeable.

La détermination du sexe des embryons bovins n'est envisageable qu'à l'âge où s'effectue le transfert, soit 7 à $8 j$ de gestation, âge lui-même conditionné par le fait qu'il s'agit du stade optimum pour la congélation de l'embryon en vue de sa conservation (Heyman, 1988).

Les premières tentatives de sexage des embryons de mammifères avant leur transfert remontent aux années 1980 . Elles étaient basées sur les possibilités offertes par l'analyse chromosomique (Picard et al, 1985), ou sur la détection immunologique d'une molécule de membrane, l'antigène $\mathrm{H}-\mathrm{Y}$, considéré comme étant spécifiquement exprimé par les cellules mâles (Wachtel et al, 1988; Booman et al, 1989).

La difficulté des techniques cytogénétiques réside dans le fait que même en sacritiant la moitié d'un embryon le nombre de cellules en division reste faible et ne permet de déterminer le sexe que dans $60 \%$ des cas. De plus, l'hemisection réduit considérablement les chances de développement ultérieur de la moitié congelée. Les essais de sexage réalisés à partir de biopsies de 10 à 20 cellules permettent un diagnostic dans seulement $30 \%$ des cas (Picard et al, 1985). Néanmoins, l'intérêt de l'analyse cytogénétique réside dans le fait qu'elle fournit un résultat fiable à $100 \%$. La technique de détection de l'antigène $\mathrm{H}-\mathrm{Y}$ ne semble pas être suffisamment performante pour être utilisée en routine chez les bovins.

Face à ces difficultés, nous avons envisagé une nouvelle approche du sexage des embryons bovins. Les résultats obtenus sur l'organisation moléculaire des chromosomes sexuels humains et murins (Bishop et al, 1985) nous ont conduits à produire des sondes moléculaires spécifiques du chromosome $Y$ des bovins. Pour ce faire, nous avons construit une bibliothèque génomique bovine enrichie en séquences mâles par la technique d'hybridation-sélection (Lamar et Palmer, 1984). Parmi les clones analysés, l'un d'entre eux BC 1.2 (brevet $n^{\circ} 8602811$ ) a fait l'objet d'une analyse approfondie afin de pouvoir être utilisé pour le sexage des embryons.

\section{MATÉRIEL ET MÉTHODES}

\section{Extraction de l'ADN}

L'ADN est extrait à partir de lymphocytes du sang périphérique provenant d'animaux mâles et femelles bovins.

\section{Blots génomiques, hybridation}

Tous les blots génomiques sont préparés à partir de $10 \mu \mathrm{g}$ d'ADN totalement digérés par les différentes enzymes de restriction utilisées ( 6 unités/ $\mu \mathrm{g}$ d'ADN). Les fragments sont séparés sur des gels d'agarose $0,8 \%(w / v)$ et transfé- 
rés sur des membranes de nylon (Southern, 1979).

Les blots ainsi obtenus sont préhybridés pendant $4 \mathrm{~h}$ à $42^{\circ} \mathrm{C}$ dans un tampon contenant $50 \%$ ( $v / v)$ de formamide desionisée, $5 \times$ Denhardt, 5 $x$ SSC $(1 \times$ SSC $=0,15 \mathrm{M} \mathrm{NaCl}, 0,015 \mathrm{M}$ citrate de sodium, $\mathrm{pH} 7,4) 0,02 \mathrm{M}$ phosphate de sodium, $\mathrm{pH} 6,7,0,5 \%$ (w/v) SDS et $100 \mu \mathrm{g} / \mathrm{ml}$ d'ADN de sperme de saumon, puis hybridés en présence de $4 \times 10^{6} \mathrm{cpm} / \mathrm{ml}$ de sonde radioactive marquée au ${ }^{32} \mathrm{P}$.

Après l'hybridation, les membranes sont lavées pendant $15 \mathrm{~min}$ à dans un tampon $2 \times$ SSC, $0,1 \%$ SDS puis deux fois $30 \mathrm{~min}$ en $0,2 \times$ SSC, $0,1 \%$ SDS $65^{\circ} \mathrm{C}$. Les autoradiographies sont effectuées sur des films Kodak XAR-5 exposés à $-70^{\circ} \mathrm{C}$ en présence d'écrans intensifiants pendant 1 à $48 \mathrm{~h}$.

\section{Synthèse et marquage des oligonucléotides}

Tous les oligonucléotides de synthèse sont marqués en extrémité $5^{\prime}$ avec du $\boldsymbol{\gamma}^{32 \mathrm{p}}$ adenosine triphosphate à l'aide de l'enzyme T4 polynucléotide kinase.

\section{Dot-blots}

Des différents échantillons d'ADN sont dénaturés avec $0,4 \mathrm{~N} \mathrm{NaOH}, 25 \mathrm{mM}$ EDTA pendant 15 min à température ambiante puis déposés à l'aide d'un appareil à dot sur des membranes de nylon. Les filtres sont neutralisés dans du tampon phosphate $1,5 \mathrm{M}, \mathrm{pH} 5,5$, pendant 2 fois 10 min.

\section{Embryons}

Les embryons sont collectés à 7-8 j de gestation, à partir de génisses superovulées. Du milieu PBS (phosphate buffered saline) contenant $1 \%$ de SAB (serum albumine bovine) est utilisé pour rincer les cornes utérines. Les embryons sont ensuite transférés dans du milieu PBS additionné de $10 \%$ de SVF (serum de veau fœtal) où ils sont disséqués grâce à l'utilisation de micromanipulateurs. Les biopsies embryonnaires sont rincées dans du PBS seul, puis déposées soit sur une lame histologique en vue de leur analyse par hybridation in situ, soit dans un tube de $0,6 \mathrm{ml}$ contenant $20 \mu \mathrm{l}$ d'eau distillée stérile en vue de l'amplification enzymatique (PCR). Dans ce dernier cas, les tubes sont immédiatement plongés dans l'azote liquide puis stockés à $-80^{\circ} \mathrm{C}$.

\section{Hybridation in situ}

Les lymphocytes bovins ou les biopsies embryonnaires sont fixées sur des lames histologiques avec une solution de Carnoy (éthanol, chloroforme, acide acétique, 6:3:1).

Un fragment de 350 paires de bases du plasmide pUC9 contenant l'insert BC 1.2 est utilisé comme sonde. Le marquage de la sonde s'effectue par nick-translation avec de I'UTP biotiné. La sonde marquée est diluée dans le liquide d'hybridation (4 x SSC, $1 \mathrm{mM}$ EDTA, $25 \mathrm{mM}$ Tris pH 7,3; $5 \times$ Denhardt, $10 \%$ dextran, $50 \%$ formamide) et déposée sur chaque lame. Les ADN sont dénaturés à $100^{\circ} \mathrm{C}$ pendant 10 min puis hybridés à $42^{\circ} \mathrm{C}$ pendant 4 à $16 \mathrm{~h}$.

Après l'hybridation, les lames sont lavées dans $2 \times$ SSC, $0,1 \times$ SSC puis dans du PBS, avant d'être incubées $1 \mathrm{~h}$ à $37^{\circ} \mathrm{C}$ avec un anticorps de chèvre antibiotine. Elles sont de nouveau lavées puis incubées avec un anticorps de lapin antichèvre couplé à la peroxydase.

Après les lavages avec le PBS, les lames sont incubées avec $10 \mu \mathrm{g} / \mathrm{ml}$ de diaminobenzidine pendant 5 à $8 \mathrm{~min}$. Pour amplifier le précipité de la diaminobenzidine, les lames sont traitées successivement avec le chloroaurate de sodium, le sulfate de sodium puis le nitrate d'argent (Burns et al, 1985). Entre chaque traitement, les lames sont soigneusement rinçées avec de l'eau distillée pour éviter la précipitation non spécifique de l'argent. Elles sont ensuite séchées et montées pour l'observation au microscope optique.

\section{Amplification enzymatique dirigée de l'ADN et des cellules}

L'ADN ou les cellules sont placés dans des tubes siliconés de $0,6 \mathrm{ml}$ sous un volume de $50 \mu$ puis introduit dans l'appareil DNA Thermal Cycler (Perkin Elmer Cetus). Après $10 \mathrm{~min}$ à $95^{\circ} \mathrm{C}$, chaque échantillon reçoit $50 \mu$ d'un mélange réactionnel contenant $200 \mu \mathrm{M}$ de chaque desoxynucléotide, $0,5 \mu \mathrm{M}$ de chacun des oligonucléotides-amorces, 4 unités d'enzyme Taq ADN polymérase, $50 \mathrm{mM} \mathrm{KCl}, 10 \mathrm{mM}$ Tris- $\mathrm{HCl}$, $\mathrm{pH} 8,3,7 \mathrm{mM} \mathrm{MgCl}_{2}, 0,01 \%$ de gélatine.

Chaque tube reçoit ensuite $100 \mu \mathrm{l}$ d'huile minérale pour éviter l'évaporation, et est soumis à 40 cycles d'amplification. Chaque cycle comprend 3 étapes : $1 \mathrm{~min}$ à $95^{\circ} \mathrm{C}, 1 \mathrm{~min}$ à $60^{\circ} \mathrm{C}, 1$ min à $72^{\circ} \mathrm{C}$ (Saiki et al, 1985). 


\section{RÉSULTATS}

\section{Obtention et caracterisation de la sonde spécifique du chromosome $Y$}

A partir de la banque génomique bovine enrichie en séquences spécifiques du mâle, nous avons obtenu 200 clones recombinants dont 131 contenant un insert. Tous ces inserts ont été analysés par l'hybridation des blots génomiques contenant de I'ADN mâle et femelle. Trois types de profil d'hybridation ont été observés : 1) non spécifique du sexe (121 inserts); 2) partiellement spécifique du mâle ( 8 inserts); 3) entièrement spécifique du mâle (2 inserts) .

Un insert dénommé $B C 1.2$ spécifique du génome mâle a été séquencé et analysé en détail.

L'hybridation in situ sur chromosomes en métaphase (Popescu et al, 1988) et l'utilisation des hybrides somatiques hamster-bovins ont permis de localiser la séquence $B C 1.2$ sur le chromosome $Y$ du taureau. D'autre part cette sonde s'est révélée uniquement spécifique des animaux du genre Bos et Bison, répétée environ 2000 fois dans le génome mâle et non transcrite.

\section{Hybridation in situ}

Cette technique appliquée à la cellule embryonnaire en interphase permet d'identifier l'hybridation de la sonde BC 1.2 au niveau de chaque noyau. Nous avons utilisé la sonde $\mathrm{BC} 1.2$ marquée à la biotine suivi d'une révélation immunocytochimique.

Dans un premier temps, nous avons testé cette technique sur les cellules lymphocytaires provenant de taureau et de vache. Dans le cas des lymphocytes du taureau, nous avons obtenu un marquage du noyau dans $85 \%$ des cellules contre $8 \%$ de marquage avec des lymphocytes femelles. II semble que dans certaines cellules mâles l'ADN ne soit pas accessible à la sonde soit à cause de l'état physiologique de la cellule (condensation en relation avec les différentes phases du cycle cellulaire), soit à cause d'une dénaturation insuffisante de I'ADN. Pour les cellules femelles il s'agit d'une précipitation non spécifique survenue au cours des différentes étapes immunocytochimiques constituant le bruit de fond inhérent à la technique. Ces deux valeurs représentent les limites de précision de l'hybridation in situ.

Après la microdissection de l'embryon, la biopsie de 10 à 20 cellules est sexée par l'hybridation avec la sonde BC 1.2 biotinée. Les résultats du sexage ont été comparés avec ceux de l'analyse cytogénétique effectuée sur la partie restante de l'embryon (environ 100 à 120 cellules) (fig 1).

Sur 19 résultats de sexage par hybridation in situ, 18 ont été corroborés par l'analyse cytogénétique. L'application de cette technique sur un nombre important d'échantillons révèle les limites de son utilisation en routine. Environ $38 \%$ des biopsies ont été perdues pendant les diverses étapes de l'hybridation et de la révélation. D'autre part, $34 \%$ des biopsies n'ont pas pu être correctement interprétées soit à cause de l'importance du bruit de fond, soit de l'état physique de la biopsie. Parmi les embryons sexés, 9 ont été transférés chez des vaches receveuses et 6 gestations ont été obtenues. Le sexe des fœetus déterminé in visu à $60 \mathrm{j}$ s'est révélé correct dans 5 cas sur 6 .

\section{Amplification enzymatique dirigée}

A partir de la séquence $B C 1.2$, nous avons déterminé celle des amorces et des 

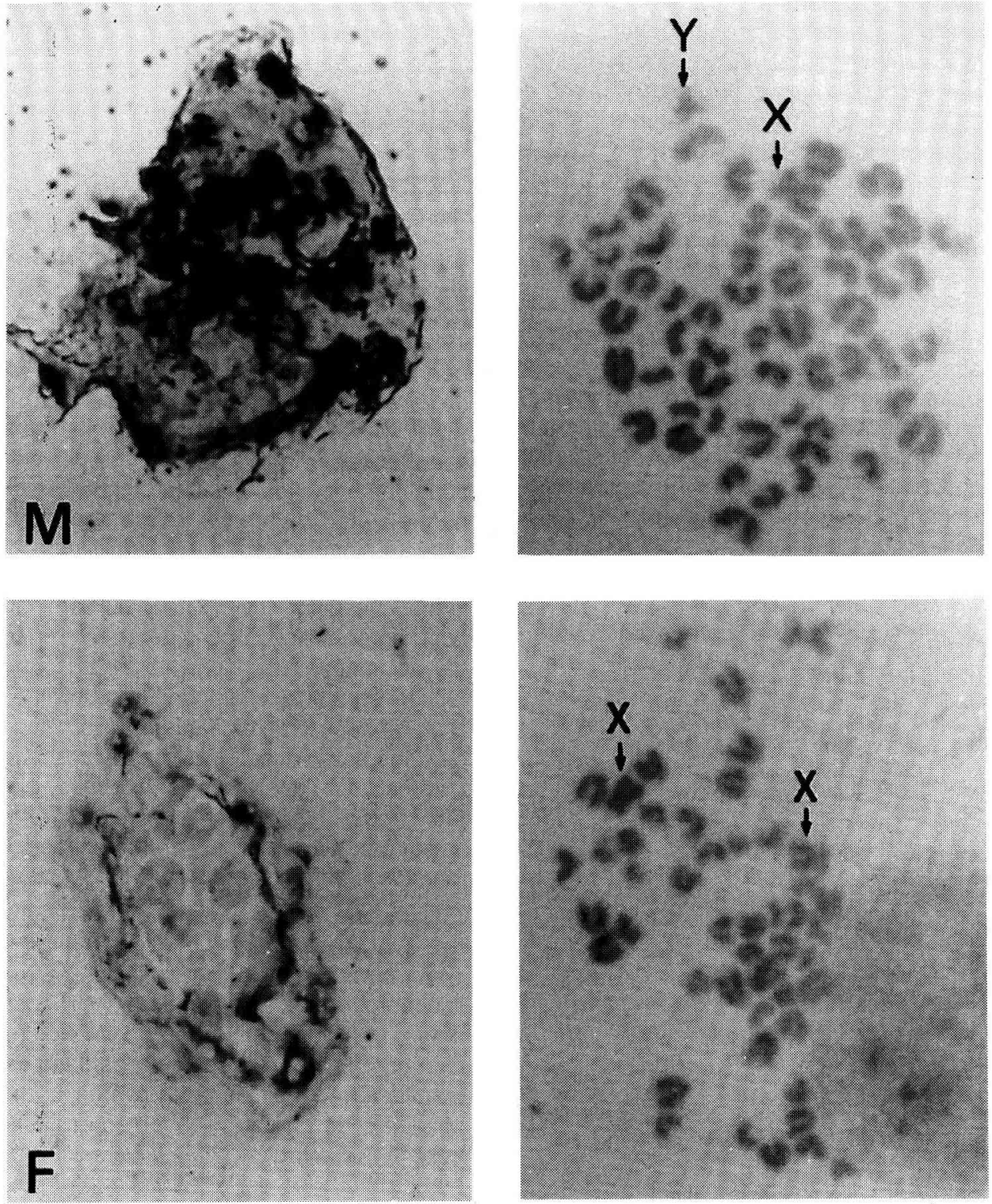

Fig 1. Résultats d'hybridation in situ avec la sonde BC 1.2 biotinée sur les biopsies embryonnaires de 10-20 cellules et la comparaison avec les caryotypes obtenus à partir du reste de l'embryon (environ 120 cellules). Le signal d'hybridation visible sur le noyau de chaque cellule correspond à la présence du chromosome $Y$ d'un embryon mâle $(M)$ alors que l'absence de ce signal correspond à un embryon femelle $(F)$. 
sondes pour leur application au sexage des biopsies embryonnaires. Nous avons mis au point les conditions d'amplification sur l'ADN génomique purifié du taureau et de la vache correspondant à un très faible nombre de cellules (50 à 200 pg d'ADN correspondant à 10-40 cellules). Après 40 cycles d'amplification, l'hybridation avec la sonde radiomarquée nous a permis d'obtenir un fort signal d'hybridation seulement avec l'ADN mâle.

Afin de démontrer la faisabilité du test directement à partir d'un faible nombre de cellules, une série de 10 échantillons contenant chacun de 50 à 70 cellules embryonnaires a été soumise à l'amplification enzymatique. Nous avons observé un fort signal d'hybridation dans le cas de 4 échantillons et aucun signal pour les 6 autres (fig 2). Par comparaison avec les résultats obtenus à partir d'ADN lymphocytaire mâle et femelle, nous avons attribué

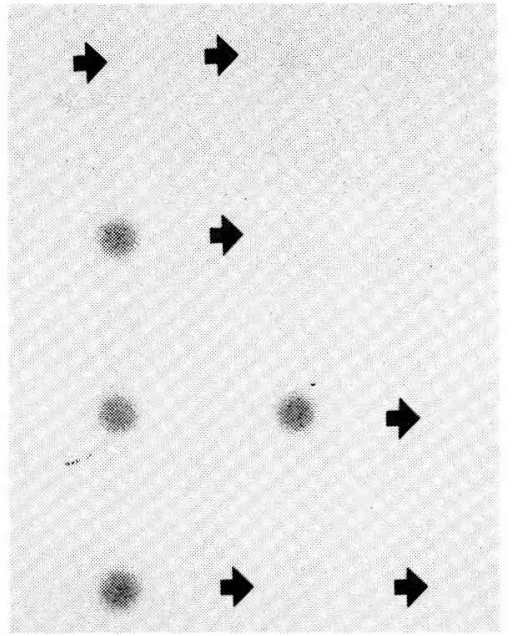

Fig 2. Résultats d'hybridation de la sonde radiomarquée sur le dot-blot contenant 10 échantillons de 50 à 70 cellules embryonnaires après 40 cycles d'amplification enzymatique. Ce signal d'hybridation correspond aux embryons mâles (présence du chromosome $Y$ ). Les flèches indiquent le dépôt des embryons déterminés comme femelles. l'obtention du signal d'hybridation à la présence du chromosome $Y$.

\section{DISCUSSION}

Grâce à l'obtention de sondes d'ADN spécifiques du chromosome $Y$ dés bovins, il est désormais possible de déterminer le sexe des embryons par hybridation moléculaire (Leonard et al, 1987; Bondioli et al, 1989). Les deux techniques décrites ici permettent d'effectuer le diagnostic à partir de prélèvements contenant seulement 10 à 50 cellules. L'hybridation in situ sur biopsies nécessite la mise en cuvre de réactions immunocytochimiques complexes qui permettent d'augmenter et de rendre visible le signal d'hybridation au niveau de chaque noyau cellulaire. L'amplification enzymatique dirigée (PCR) est utilisée pour augmenter de façon considérable la quantité de matériel de départ et rendre la détection du signal d'hybridation plus aisée. Bien qu'il soit indispensable de microdisséquer les embryons, il n'est pas nécessaire, comme pour l'analyse cytogénétique, d'effectuer des cultures cellulaires ni de travailler à partir de cellules en métaphase; le diagnostic est donc réalisable en 24-48 h.

Chacune des deux techniques présente des avantages et des inconvénients qui lui sont propres. La possibilité d'automatisation offerte par la technique PCR constitue un progrès notable pour le traitement d'un grand nombre d'échantillons dans des conditions normalisées, tandis que la manipulation de nombreuses lames en séries apparaît comme délicate. De plus, les étapes multiples de la révélation immunocytochimique sont peu adaptables à une utilisation de la technique d'hybridation in situ en routine. Le problème de la sensibilité se pose ici par défaut, à l'inverse de la technique PCR ou l'extrême sensibilité peut être l'inconvénient majeur de la mé- 
thode. Des études récentes ont montré qu'il était désormais possible d'appliquer la technique PCR à une seule cellule ( $\mathrm{Li}$ et al, 1988; Handyside et al, 1989); cette évolution technique est souhaitable, car moins l'embryon sera endommagé, meilleure sera sa viabilité ultérieure. De plus, le fait d'utiliser le minimum de cellules permettra d'étudier à partir d'un même embryon plusieurs gènes marqueurs et de ce fait sélectionner les animaux les plus performants pour leurs compétences zootechniques (résistance aux maladies, haut potentiel génétique). Cependant, il faut souligner que le fait de travailler à partir de très petites quantités de matériel ( 1 à 10 cellules) nécessite un grand nombre de cycles d'amplification, ce qui est la cause de nombreuses complications telles que : l'amplification artéfactuelle des amorces ou de I'ADN ainsi que l'augmentation des risques de contamination par I'ADN amplifié. Ces problèmes peuvent être minimisés en prenant de grandes précautions lors de la manipulation des échantillons. La séparation physique lors du traitement des tubes avant et après l'amplification devient alors indispensable.

\section{CONCLUSION}

Les résultats présentés ici montrent qu'il est possible de déterminer le sexe des embryons bovins à partir d'une biopsie à l'aide de sondes moléculaires par hybridation in situ ou l'amplification enzymatique. Bien qu'importante sur le plan historique, la technique d'hybridation in situ présente les inconvénients inhérents à la manipulation de quelques cellules déposées sur une lame de verre et à l'utilisation d'une succession de réactions complexes. D'autre part, il est difficile d'envisager son automatisation, seule possibilité d'obtenir une bonne reproductivité et une application à grande échelle. Appliquée à notre projet, la technique PCR a déjà permis l'obtention de résultats tout à fait encourageants à partir de 50 à 70 cellules embryonnaires. Les perfectionnements possibles devraient conduire à un sexage efficace et automatisable à partir d'un nombre plus restreint de cellules.

\section{REMERCIEMENTS}

Nous remercions $Y$ Heyman, $P$ Chesné et MG Stinakre pour la collecte et la microdissection des embryons, Mme Hors-Cayla pour avoir mis à notre disposition les lignées d'hybrides somatiques hamster-bovins. Ce travail a été partiellement financé par un contrat ANVAR $n^{\circ} 3517 \mathrm{~A}$.

\section{RÉFÉRENCES}

Bishop C, Weissenbach J, Casanova M, Bernheim A, Fellous M (1985) DNA sequences and analysis of the human $Y$ chromosome. In: The Y Chromosome, part A. (Sandberg AA, ed) Allan R Liss Inc, New York, 141-176

Bondioll KR, Ellis SB, Pryor JH, Williams MW, Harpold MM (1989) The use of male specific chromosomal DNA fragments to determine the sex of bovine preimplantation embryos. Theriogenology 31, 95-103

Booman P, Kruijt L, Veerhuis R, Hengst AM, Tieman M, Ruch FE (1989) Sexing bovine embryos with monoclonal antibodies against the H-Y antigen. Livestock Prod Sci 23, 1-16

Burns J, Chan VTW, Jonasson J, Fleming K, Taylor S, McGee JOD (1985) Sensitive system for visualising biotinylated DNA probes hybridised in situ: rapid sex determination of intact cells. J Clin Pathol 38, 1085-1092

Handyside AH, Penketh RJA, Winston RML, Pattinson JK, Delhanty JDA, Tuddenham EGD (1989) Biopsy of human preimplantation embryos and sexing by DNA amplification. Lancet $\mathrm{i}, 347-349$

Heyman $Y$ (1988) Moment de la transplantation et succès de la gestation chez les mammifères. Reprod Nutr Dev 28, 1773-1780

Lamar EE, Palmer E (1984) Y encoded, species-specific DNA in mice. Cell 37, 171-177 
Leonard M, Kirszenbaum M, Cotinot C, Chesne $P$, Heyman $Y$, Stinnakre $M$, Bishop C, Vaiman M, Fellous M (1987) Sexing bovine embryos using $Y$ chromosome specific DNA probe. Theriogenology 27, 248

Li H, Gyllensten UB, Cui X, Saiki RK, Erlich HA, Arnheim N (1988) Amplification and analysis of DNA sequences in single human sperm and diploid cells. Nature 335, 414-417

Picard L, King WA, Betteridge KJ (1985) Production of sexed calves from frozen-thawed embryos. Vet Rec 117, 603-608

Popescu CP, Cotinot C, Bosher J, Kirszenbaum $M$ (1988) Chromosomal localization of a bovine male specific probe. Ann Genet 31, 39-42

Saiki RK, Scharf S, Faloona F, Mullis KB, Horn GT, Erlich HA, Arnheim N (1985) Enzymatic amplification of $\beta$-globin genomic sequences and restriction site analysis for diagnosis of sickle cell anemia. Science 230, 1350-1354

Southern E (1979) Gel electrophoresis of restriction fragments. Methods Enzymol 68, 152176

Wachtel SS, Nakamura D, Wachtel G, Felton W, Kent M, Jaswaney $V$ (1988) Sex selection with monoclonal $\mathrm{H}-\mathrm{Y}$ antibody. Fertil Steril $50,355-360$ 